\title{
Turbulent mixing and wave radiation in non-Boussinesq internal bores
}

\author{
Borden, Zac; Koblitz, Tilman; Meiburg, Eckart
}

Published in:

Physics of Fluids

Link to article, DOI:

$10.1063 / 1.4745478$

Publication date:

2012

Document Version

Publisher's PDF, also known as Version of record

Link back to DTU Orbit

Citation (APA):

Borden, Z., Koblitz, T., \& Meiburg, E. (2012). Turbulent mixing and wave radiation in non-Boussinesq internal bores. Physics of Fluids, 24(8), Paper 082106. https://doi.org/10.1063/1.4745478

\section{General rights}

Copyright and moral rights for the publications made accessible in the public portal are retained by the authors and/or other copyright owners and it is a condition of accessing publications that users recognise and abide by the legal requirements associated with these rights.

- Users may download and print one copy of any publication from the public portal for the purpose of private study or research.

- You may not further distribute the material or use it for any profit-making activity or commercial gain

- You may freely distribute the URL identifying the publication in the public portal

If you believe that this document breaches copyright please contact us providing details, and we will remove access to the work immediately and investigate your claim. 


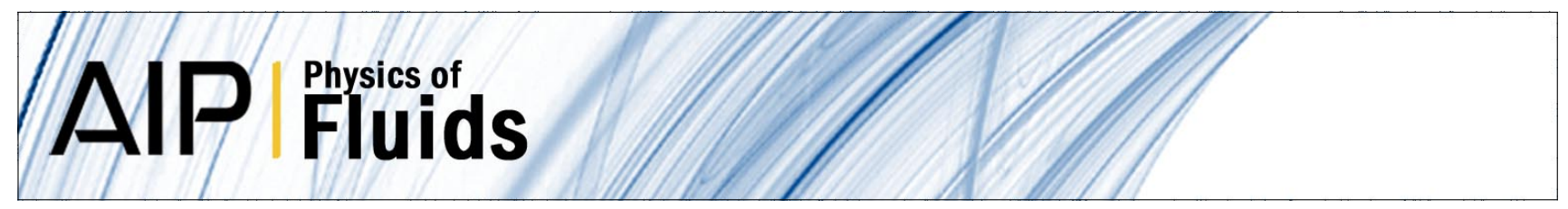

\section{Turbulent mixing and wave radiation in non-Boussinesq internal bores}

Zac Borden, Tilman Koblitz, and Eckart Meiburg

Citation: Phys. Fluids 24, 082106 (2012); doi: 10.1063/1.4745478

View online: http://dx.doi.org/10.1063/1.4745478

View Table of Contents: http://pof.aip.org/resource/1/PHFLE6/v24/i8

Published by the American Institute of Physics.

\section{Related Articles}

Reduction of mean-square advection in turbulent passive scalar mixing

Phys. Fluids 24, 075104 (2012)

Modeling molecular mixing in a spatially inhomogeneous turbulent flow

Phys. Fluids 24, 025103 (2012)

Three-dimensionalization of the stratified mixing layer at high Reynolds number

Phys. Fluids 23, 111701 (2011)

Confocal imaging of laminar and turbulent mixing in a microscale multi-inlet vortex nanoprecipitation reactor Appl. Phys. Lett. 99, 204103 (2011)

Growth of a Richtmyer-Meshkov turbulent layer after reshock

Phys. Fluids 23, 095107 (2011)

\section{Additional information on Phys. Fluids}

Journal Homepage: http://pof.aip.org/

Journal Information: http://pof.aip.org/about/about_the_journal

Top downloads: http://pof.aip.org/features/most_downloaded

Information for Authors: http://pof.aip.org/authors

\section{ADVERTISEMENT}

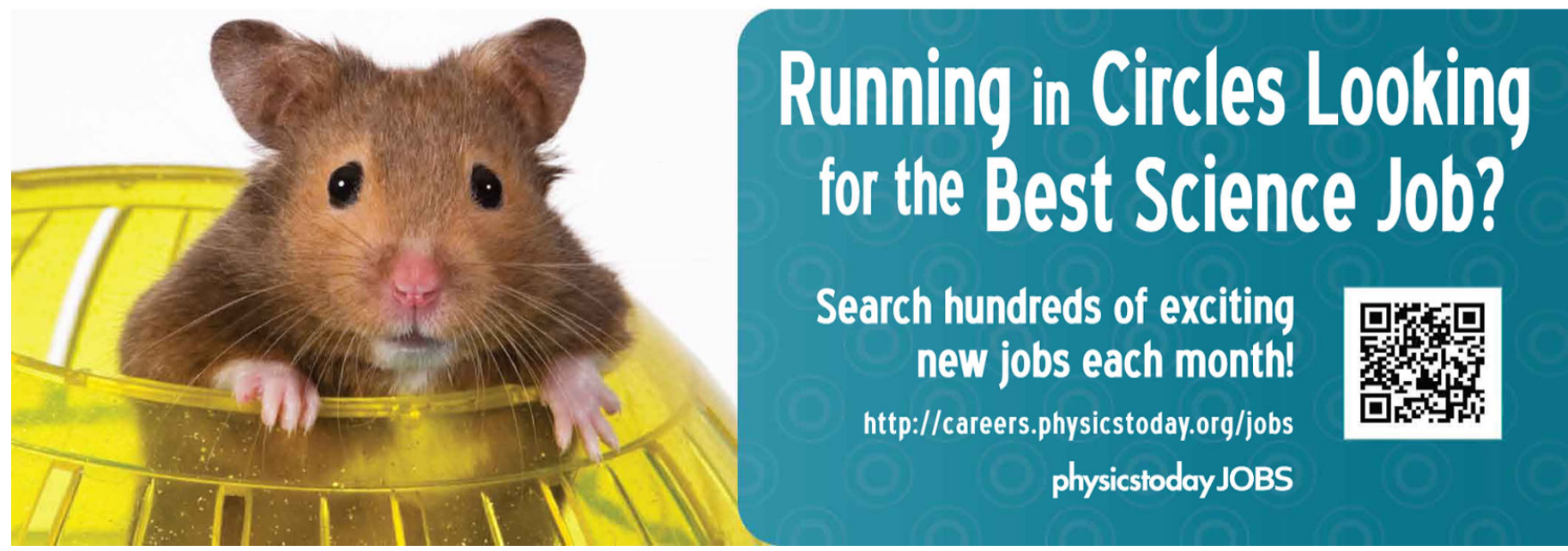




\title{
Turbulent mixing and wave radiation in non-Boussinesq internal bores
}

\author{
Zac Borden, ${ }^{1}$ Tilman Koblitz, ${ }^{1,2}$ and Eckart Meiburg ${ }^{1}$ \\ ${ }^{1}$ Department of Mechanical Engineering, University of California at Santa Barbara, \\ Santa Barbara, California 93106, USA \\ ${ }^{2}$ Ris $\phi$ National Laboratory for Sustainable Energy, DK-4000 Roskilde, Denmark
}

(Received 28 April 2012; accepted 26 July 2012; published online 16 August 2012)

\begin{abstract}
Bores, or hydraulic jumps, appear in many natural settings and are useful in many industrial applications. If the densities of the two fluids between which a bore propagates are very different (i.e., water and air), the less dense fluid can be neglected when modeling a bore analytically - a single-layer hydraulic model will accurately predict a bore's speed of propagation. A two-layer model is required, however, if the densities are more similar. Mass is conserved separately in each layer and momentum is conserved globally, but the model requires for closure an assumption about the loss of energy across a bore. In the Boussinesq limit, it is known that there is a decrease of the total energy flux across a bore, but in the expanding layer, turbulent mixing at the interface entrains high speed fluid from the contracting layer, resulting in an increase in the flux of kinetic energy across the expanding layer of a bore. But it is unclear if this finding will extend to non-Boussinesq bores. We directly examine the flux of energy within non-Boussinesq bores using two-dimensional direct numerical simulations and find that a gain of energy across the expanding layer only occurs for bores where the density ratio, defined as the ratio of the density of the lighter fluid to the heavier fluid, is greater than approximately one half. For smaller density ratios, undular waves generated at the bore's front dominate over the effects of turbulent mixing, and the expanding layer loses energy across the bore. Based on our results, we show that if one can predict the amount of energy radiated by bores through undular waves, it is possible to derive an accurate model for the propagation of non-Boussinesq bores. () 2012 American Institute of Physics. [http://dx.doi.org/10.1063/1.4745478]
\end{abstract}

\section{INTRODUCTION}

Bores, otherwise known as hydraulic jumps, are flow phenomena in which a sharp rise in height propagates along a horizontal interface between two fluids of different densities. They appear in natural geophysical flows and are also important in industrial applications. The difference in densities between the fluids on either side of the horizontal interface, described by the density ratio $\gamma=\rho_{2} / \rho_{1}$, where $\rho_{2}$ is the density of the lighter fluid, and $\rho_{1}$ the density of the heavier, significantly affects the propagation and behavior of a bore. Historically, most research has focused on bores with density ratios either very close to zero or very close to one because most — but not all —natural and industrial bores fall into one of these two categories. The propagation of bores with a near zero density ratio, such as tidal bores ${ }^{1}$ and engineered jumps in dam spillways, ${ }^{2}$ is well described analytically by a single-layer hydraulic model that conserves mass and momentum fluxes in the more dense fluid across the front of the bore. ${ }^{3}$

Bores with a density ratio very close to one are called internal bores. Internal bores can form in the atmosphere by the interaction of a sea breeze with a temperature inversion layer, ${ }^{4,5}$ or by the interaction of a thunderstorm outflow with an inversion layer. ${ }^{6}$ They also occur in marine environments by the breaking of internal waves, ${ }^{7}$ the interaction of tides with ocean floor topography, ${ }^{8,9}$ or the flow of turbidity currents past submarine obstacles. ${ }^{10-12}$ 


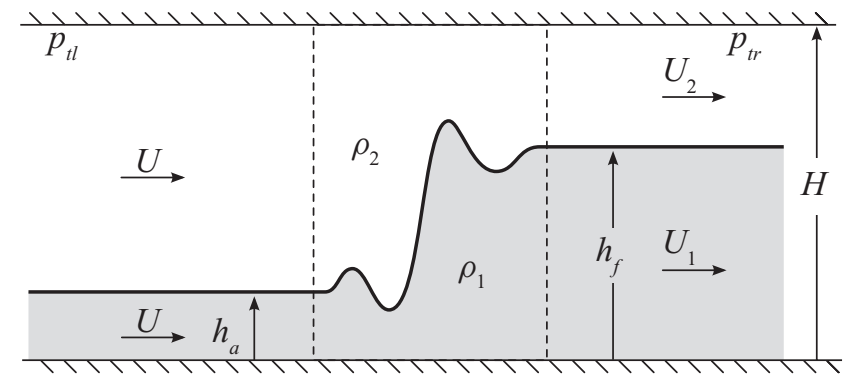

FIG. 1. Idealized geometry and flow of a two-layer bore in a reference frame moving with the front of the bore.

To describe an internal bore analytically, a two-layer hydraulic model is typically used. Within the simplified flow in Figure 1, mass is conserved separately in each layer and the global change in momentum flux across the bore is balanced by a change in pressure. Because the density difference across the interface is small—on the order of $\gamma \approx 0.97$ in the above examples-the Boussinesq approximation is also invoked.

Unlike a single-layer hydraulic model, a two-layer model of internal bores requires a jump condition relating the upstream and downstream energy fluxes in order to produce a solution for the bore's propagation velocity as a function of the bore's height and the channel's depth. Finding the correct energy jump condition has been the focus of several research studies. Wood and Simpson ${ }^{13}$ (WS) first argued for a conservation of energy in the upper, contracting layer of a bore based on the mixing of dye streaks in laboratory experiments of internal bores. Klemp et al. ${ }^{14}$ (KRS) however, obtained better agreement with available laboratory scale experimental data if they instead imposed energy conservation on the lower, expanding layer. It was commonly assumed that these two scenarios represented the limits of a bore's speed of propagation, and that real bores fell somewhere in between. Very recently, however, high-resolution numerical simulations by Borden et al. ${ }^{15}$ found that turbulent mixing at the interface between the two layers causes the lower, expanding layer of a bore to gain a small amount of energy across the front. Based on this observation, they developed a new model for predicting the velocity of Boussinesq bores.

Not all bores fall into the categories of a single-layer hydraulic jump or a Boussinesq internal bore. For example, in the petroleum industry, T-junctions in pipe flows are often used to separate two-phase flows of oil and water. ${ }^{16}$ Roberts and Hibberd ${ }^{17}$ found that internal hydraulic jumps can form in these T-junctions and inhibit phase separation. In these internal jumps, the density ratio can range from $\gamma=0.4$ to $\gamma=0.9$. The Boussinesq approximation is not applicable, but the upper layer is too dense to neglect. Previous numerical simulations of gravity currents, which share features with internal bores, by Birman et al..$^{18}$ show that the propagation velocity of non-Boussinesq gravity currents with a non-zero density ratio is not accurately predicted by either single-layer or Boussinesq two-layer models. The current's height, propagation velocity, and global energy dissipation were strongly dependent on the density ratio. Researchers have recently developed non-Boussinesq analytical models of gravity currents that include this dependence, ${ }^{19-21}$ but to our knowledge, no two-layer, non-Boussinesq models exist for internal bores.

In this paper, we develop such a model through a process similar to Borden et al. ${ }^{15}$ First, we develop a framework that provides a non-Boussinesq bore's front velocity given the change in energy flux across the bore in either layer and some geometrical parameters (Sec. II). Next, we simulate laboratory scale internal bores using direct numerical simulation, described in Sec. III, and measure their front velocities. Entering these front velocities into our framework predicts the change in energy flux that must be occurring in each layer. We then search for the mechanisms that explain the change in energy flux. In particular, we investigate the effects of turbulent mixing in Sec. IV A and wave generation and breaking in Sec. IV B. We also show how these effects can be included in a simplified model for predicting the dynamics of non-Boussinesq bores. 


\section{ANALYTICAL FRAMEWORK FOR NON-BOUSSINESQ INTERNAL BORES}

To develop an analytical model for non-Boussinesq internal bores, we require a framework that provides the front velocity as a function of the change in energy flux across a bore in either layer. For Boussinesq internal bores, an appropriate framework was derived by Li and Cummins, ${ }^{22}$ which we hereafter refer to as the LC relation. The LC relation is a two-layer hydraulic model that assumes mass conservation in each layer, a global balance of momentum, and an arbitrary change in energy flux in either the upper or lower layer. Here, we extend the LC relation to include an arbitrary density ratio.

Consider the idealized bore in Figure 1. In a reference frame moving with the bore's front, conservation of mass in a control volume spanning the front and encompassing either layer produces

$$
\begin{gathered}
U_{1} h_{f}=U h_{a}, \\
U_{2}\left(H-h_{f}\right)=U\left(H-h_{a}\right) .
\end{gathered}
$$

The LC relation also states that the change in global momentum flux is balanced by the pressure. In general, this relation is represented by

$$
\int_{0}^{H}\left(p_{l}+\rho U^{2}\right) \mathrm{d} z=\int_{0}^{H}\left(p_{r}+\rho u_{r}^{2}\right) \mathrm{d} z
$$

where $u_{r}$ represents the local velocity far downstream of the bore's front, and $p_{l}$ and $p_{r}$ represent the hydrostatic pressure plus the pressure at the top of the channel $\left(p_{t l}\right.$ and $\left.p_{t r}\right)$ at streamwise locations far upstream and downstream of the bore's front. For the geometry in Figure 1, we can expand Eq. (3) as

$$
\begin{aligned}
& \rho_{2} U^{2}\left(H-h_{a}\right)+\rho_{1} U^{2} h_{a}+p_{t l} H+\frac{1}{2} \rho_{2} g\left(H-h_{a}\right)^{2}+\rho_{2} g\left(H-h_{a}\right) h_{a}+\frac{1}{2} \rho_{1} g h_{a}^{2} \\
= & \rho_{2} U_{2}^{2}\left(H-h_{f}\right)+\rho_{1} U_{1}^{2} h_{f}+p_{t r} H+\frac{1}{2} \rho_{2} g\left(H-h_{f}\right)^{2}+\rho_{2} g\left(H-h_{f}\right) h_{f}+\frac{1}{2} \rho_{1} g h_{f}^{2} .
\end{aligned}
$$

Combining Eqs. (1), (2), and (4) produces an expression for the pressure change across the top of the channel,

$$
\frac{p_{t r}-p_{t l}}{\rho_{1}}=\frac{\Delta p}{\rho_{1}}=\frac{h_{f}-h_{a}}{H} U^{2}\left[\frac{H\left(h_{a}-\gamma h_{f}\right)+(\gamma-1) h_{a} h_{f}}{h_{f}\left(H-h_{f}\right)}\right]+\frac{g^{\prime}}{2 H}\left(h_{a}^{2}-h_{f}^{2}\right),
$$

where $g^{\prime}=g(1-\gamma)$ is the reduced gravity. Next, we write expressions equating the energy flux in each layer across the bore,

$$
\begin{aligned}
& \frac{1}{2} \rho_{2} U^{3}\left(H-h_{a}\right)+\frac{1}{2} \rho_{2} g U\left(H^{2}-h_{a}^{2}\right)+\frac{1}{2} \rho_{2} g U\left(H-h_{a}\right)^{2}+p_{t l} U\left(H-h_{a}\right) \\
= & \frac{1}{2} \rho_{2} U_{2}^{3}\left(H-h_{f}\right)+\frac{1}{2} \rho_{2} g U_{2}\left(H^{2}-h_{f}^{2}\right)+\frac{1}{2} \rho_{2} g U_{2}\left(H-h_{f}\right)^{2}+p_{t r} U_{2}\left(H-h_{f}\right)-\dot{E}_{u}, \\
& \frac{1}{2} \rho_{1} U^{3} h_{a}+\rho_{1} g U h_{a}^{2}+p_{t l} U h_{a}+\rho_{2} g U\left(H-h_{a}\right) h_{a} \\
= & \frac{1}{2} \rho_{1} U_{1}^{3} h_{f}+\rho_{1} g U_{1} h_{f}^{2}+p_{t r} U_{1} h_{f}+\rho_{2} g U_{1}\left(H-h_{f}\right) h_{f}-\dot{E}_{l},
\end{aligned}
$$

where $\dot{E}_{u}$ and $\dot{E}_{l}$ represent any change in the kinetic energy flux across the bore in the upper and lower layers which is not captured by the work done by the horizontal pressure gradient (such as, but not limited to, viscous dissipation). Combining Eqs. (6) or (7) with the change in pressure across the top of the channel expressed in Eq. (5) and nondimensionalizing produces a non-Boussinesq 
generalization of the $\mathrm{LC}$ relation,

$$
\begin{aligned}
\dot{e}_{u} & =-\frac{(1-r)(R-1)}{2} u_{f}\left[\frac{2 R^{2} r^{2}(\gamma-1)-\gamma R^{2} r+R r(4-\gamma)-2}{R(1-R r)^{2}} u_{f}^{2}+R+1\right], \\
\dot{e}_{l} & =-\frac{R-1}{2} u_{f}\left[\frac{2 R^{2} r^{2}(1-\gamma)+R^{2} r(2 \gamma-1)-3 R r+R+1}{R^{2}(1-R r)} u_{f}^{2}+r+R r-2\right],
\end{aligned}
$$

where $r=h_{a} / H, R=h_{f} / h_{a}, u_{f}=U /\left(g^{\prime} h_{a}\right)^{1 / 2}$, and $\dot{e}=\dot{E} /\left(\rho_{1} g^{\prime 3 / 2} h_{a}^{5 / 2}\right)$. The non-Boussinesq LC relation can be used to recover non-Boussinesq versions of WS and KRS models by setting $\dot{e}_{u}$ or $\dot{e}_{l}$ equal to zero, respectively,

$$
\begin{aligned}
& u_{w s}=\left\{\frac{R(R+1)(1-R r)^{2}}{\gamma R^{2} r-\operatorname{Rr}(4-\gamma)-2 R^{2} r^{2}(\gamma-1)+2}\right\}^{1 / 2}, \\
& u_{k r s}=\left\{\frac{R^{2}(2-r-R r)(1-R r)}{2 R^{2} r^{2}(1-\gamma)+R^{2} r(2 \gamma-1)-3 R r+R+1}\right\}^{1 / 2} .
\end{aligned}
$$

Both of these equations, as well as the non-Boussinesq LC relation (Eqs. (8) and (9)), reduce to the correct Boussinesq expressions when $\gamma=1$. They are,

$$
\begin{aligned}
\dot{e}_{u_{B}} & =-\frac{(R-1)(1-r)}{2} u_{f}\left[-\frac{R^{2} r-3 R r+2}{R(1-R r)^{2}} u_{f}^{2}+1+R\right], \\
\dot{e}_{l_{B}} & =-\frac{(R-1)}{2} u_{f}\left[\frac{R^{2} r-3 R r+R+1}{R^{2}(1-R r)} u_{f}^{2}+R r+r-2\right], \\
u_{w s_{B}} & =\left\{\frac{R(1+R)(1-R r)^{2}}{R^{2} r-3 R r+2}\right\}^{1 / 2}, \\
u_{k r s_{B}} & =\left\{\frac{R^{2}[2-r(1+R)](1-R r)}{R^{2} r-3 R r+R+1}\right\}^{1 / 2} .
\end{aligned}
$$

Furthermore, we can recover the single-layer model from the non-Boussinesq WS model and the non-Boussinesq LC relation for the energy change in the lower layer by setting $\gamma=0$ and $r=0$ :

$$
\begin{aligned}
& u_{s l}=\left\{\frac{1}{2} R(R+1)\right\}^{1 / 2}, \\
& \dot{e}_{s l}=-(R-1) u_{f}\left[\frac{R+1}{2 R^{2}} u_{f}^{2}-1\right] .
\end{aligned}
$$

The KRS model, however, does not produce the single-layer front velocity or energy loss in this limit. Its underlying assumption, that all energy dissipated across a bore is dissipated in the upper layer, is inconsistent with the omission of the upper layer in the single-layer model.

The non-Boussinesq LC relation (Eqs. (8) and (9)) predicts that for front velocities near WS and KRS, there is always a global loss of energy across a bore (Figure 2(a)). In bores with front velocities between WS and KRS, the global energy loss is distributed between both layers. But in bores propagating slower than KRS, the lower layer gains energy across the bore, and in bores propagating faster than WS, the upper layer gains energy. This finding is consistent with the Boussinesq LC relation and holds regardless of the density ratio (Figure 2(b)). If we assume that a gain of energy in one fluid layer is unphysical, then the WS and KRS velocities represent bounds on a bore's speed of propagation. Klemp et al. ${ }^{14}$ however, speculated, and Borden et al. ${ }^{15}$ confirmed that a gain of energy in the lower layer is plausible because of mixing at the layers' interface. In Secs. III and IV, we will investigate whether or not this finding extends to non-Boussinesq bores. 

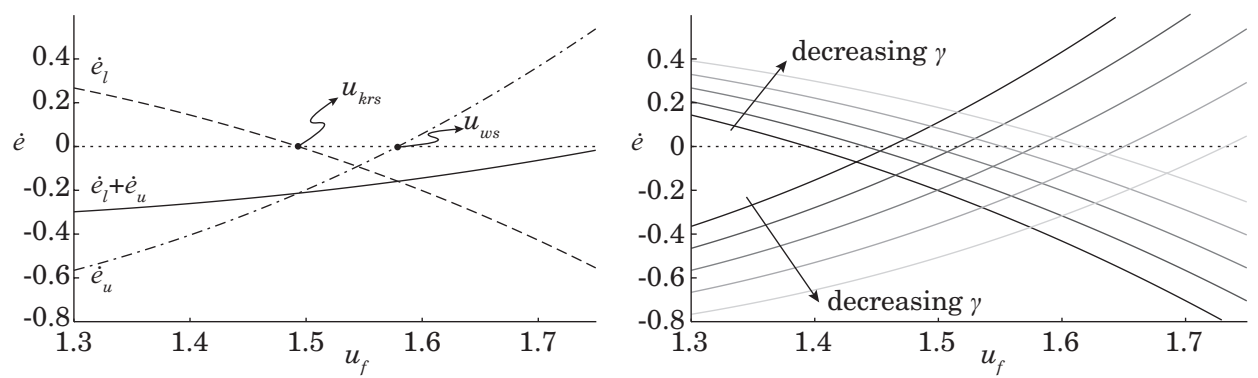

FIG. 2. (a) Energy change in each layer, as predicted by the non-Boussinesq LC relation, plotted as a function of the front velocity for $R=2, r=0.1$, and $\gamma=0.5$. The WS and KRS front velocities are recovered when $\dot{e}_{u}$ or $\dot{e}_{l}$ equal zero. (b) Energy change in the upper and lower layers for $\gamma=\{1,0.75,0.5,0.25,0\}$. Notice that for smaller density ratios, the WS and KRS bounds on the velocity become greater.

\section{NON-BOUSSINESQ SIMULATIONS}

Our two-dimensional simulations of internal bores model a dam break - a release of a rectangular volume of fluid with density $\rho_{1}$ into a two-layer channel of width $2 L$ and height $H$ (Figure 3 ). In our domain, the velocity and pressure are governed by the incompressible, non-Boussinesq NavierStokes equations, and the density evolves according to an advection-diffusion equation. For a constant kinematic viscosity $\tilde{v}$ (the tilde denotes a dimensional quantity), these equations are written as

$$
\begin{aligned}
\nabla \cdot \boldsymbol{u} & =0, \\
\rho \frac{\mathrm{D} \boldsymbol{u}}{\mathrm{D} t} & =\frac{\rho}{1-\gamma} \boldsymbol{e}_{g}-\nabla p+\frac{1}{R e} \nabla \cdot(2 \rho \mathbf{S}), \\
\frac{\mathrm{D} \rho}{\mathrm{D} t} & =\frac{1}{\operatorname{ReSc}} \nabla^{2} \rho,
\end{aligned}
$$

where $\mathrm{D} / \mathrm{D} t$ denotes the material derivative of a quantity, $\boldsymbol{u}=(u, w)^{T}$ is the velocity vector, $p$ is the local pressure, $\rho$ is the local density, $\mathbf{S}=1 / 2\left(\partial u_{i} / \partial x_{j}+\partial u_{j} / \partial x_{i}\right)$ is the rate of strain tensor, and $\boldsymbol{e}_{g}=(0,-1)^{T}$ is a unit vector that points in the direction of gravity. By neglecting the presence of the material derivative of density in the continuity equation, we are assuming that the timescale of diffusion is much longer than the timescale of advection in our domain, i.e., that the Péclet number $P e=R e S c$ is large. This common assumption is used in other simulations of non-Boussinesq miscible fluids such as in Birman et al. ${ }^{18}$ For numerical reasons, however, we still include the effects of diffusion in Eq. (20) in order to prevent discontinuities in the computation of the density field. Eqs. (18)-(20) are made dimensionless by the fluid height ahead of the bore, $\tilde{h}_{a}$, the lower layer density, $\tilde{\rho}_{1}$, the buoyancy velocity $\tilde{u}_{b}=\left(\tilde{g}^{\prime} \tilde{h}_{a}\right)^{1 / 2}$ in which $\tilde{g}^{\prime}$ is the reduced gravity, and the pressure scale $p=\tilde{p} /\left(\tilde{\rho}_{1} \tilde{u}_{b}^{2}\right)$. The two non-dimensional parameters

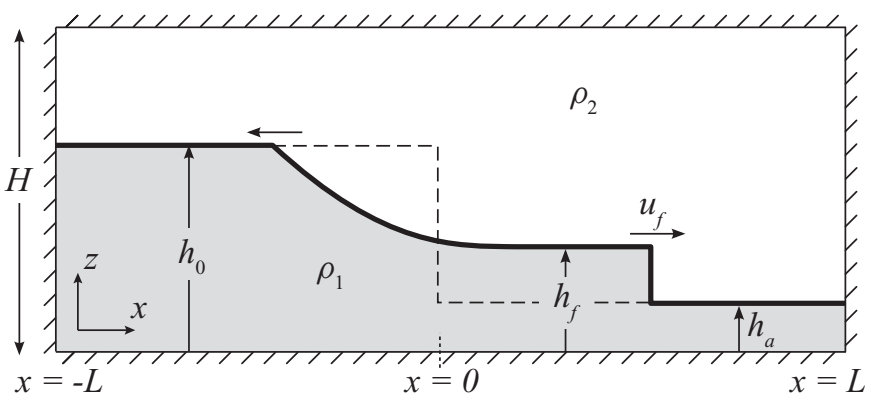

FIG. 3. Schematic of a dam break problem in a laboratory reference frame. The dashed line is the initial condition and the solid line is the state after some time has passed. 
appearing are the Reynolds number, $R e$, and the Schmidt number, $S c$, given as

$$
\operatorname{Re}=\frac{\tilde{u}_{b} \tilde{h}_{a}}{\tilde{v}}, \quad S c=\frac{\tilde{v}}{\tilde{K}},
$$

where $\tilde{K}$ is the molecular diffusivity.

If instead of using a constant kinematic viscosity, we enforce a constant dynamic viscosity, $\tilde{\mu}$, over the whole domain, Eq. (19) becomes

$$
\rho \frac{\mathrm{D} \boldsymbol{u}}{\mathrm{D} t}=\frac{\rho}{1-\gamma} \boldsymbol{e}_{g}-\nabla p+\frac{1}{R e} \nabla^{2} \boldsymbol{u} .
$$

To simulate Eqs. (18)-(20) numerically, we recast them into streamfunction-vorticity form. They become

$$
\begin{aligned}
\nabla^{2} \psi= & -\omega, \\
\frac{\mathrm{D} \omega}{\mathrm{D} t}= & \frac{1}{\operatorname{Re}} \nabla^{2} \omega-\frac{\rho_{x}}{(1-\gamma) \rho}+\frac{\rho_{z}}{\rho} \frac{\mathrm{D} u}{\mathrm{D} t}-\frac{\rho_{x}}{\rho} \frac{\mathrm{D} w}{\mathrm{D} t} \\
& +\frac{1}{\rho \operatorname{Re}}\left[2 \rho_{x} \nabla^{2} w-2 \rho_{z} \nabla^{2} u+4 \rho_{x z} w_{z}+\left(u_{z}+w_{x}\right)\left(\rho_{x x}-\rho_{z z}\right)\right], \\
\frac{\mathrm{D} \rho}{\mathrm{D} t}= & \frac{1}{\operatorname{ReSc}} \nabla^{2} \rho,
\end{aligned}
$$

where in these equations, a subscript denotes the partial derivative with respect to that direction, and the vorticity $\omega$ is a scalar quantity given by

$$
\omega=\frac{\partial w}{\partial x}-\frac{\partial u}{\partial z},
$$

and the streamfunction $\psi$ is defined as

$$
u=\frac{\partial \psi}{\partial z}, \quad w=-\frac{\partial \psi}{\partial x} .
$$

Again, if instead of enforcing a constant kinematic viscosity, we wish to have the dynamic viscosity remain constant, Eq. (23) becomes

$$
\frac{\mathrm{D} \omega}{\mathrm{D} t}=\frac{1}{\rho \operatorname{Re}} \nabla^{2} \omega-\frac{\rho_{x}}{(1-\gamma) \rho}+\frac{\rho_{z}}{\rho} \frac{\mathrm{D} u}{\mathrm{D} t}-\frac{\rho_{x}}{\rho} \frac{\mathrm{D} w}{\mathrm{D} t} .
$$

We perform the direct numerical simulation of Eqs. (22)-(24) and(27) by modifying the Boussinesq code of Borden et al. ${ }^{15}$ to simulate these non-Boussinesq governing equations. The code solves the equations on a uniform rectangular grid with a high resolution, typically on the order of $8193 \times 513$, which allows for a laboratory scale Reynolds number. Numerical discretization and integration are performed with Fourier sine or cosine expansions for streamwise discretization, sixth order compact finite differences for normal discretization, and a third order total variation diminishing (TVD) Runge-Kutta method for time integration. The local time derivatives of the velocity in Eqs. (23) and (27) are computed by first order backward extrapolation. Test calculations performed by Birman et al. ${ }^{18}$ demonstrated that the low-order accuracy in these terms did not influence the results in a measurable way.

Within this investigation, we eliminate the effect of dissipation at the boundaries by using a slip boundary condition in order to better approximate the assumptions of the analytical controlvolume models. Borden et al. ${ }^{15}$ examined the effect of the boundary condition on the propagation of Boussinesq internal bores and found no significant influence.

One potential shortcoming of our simulations is the inability of our two-dimensional code to reproduce three-dimensional flow structures and instabilities. The differences between two- and three-dimensional simulations of Boussinesq internal bores were explored by Borden et al. ${ }^{15}$ They found that global flow properties, such as front velocity and bore height, were essentially unaffected 


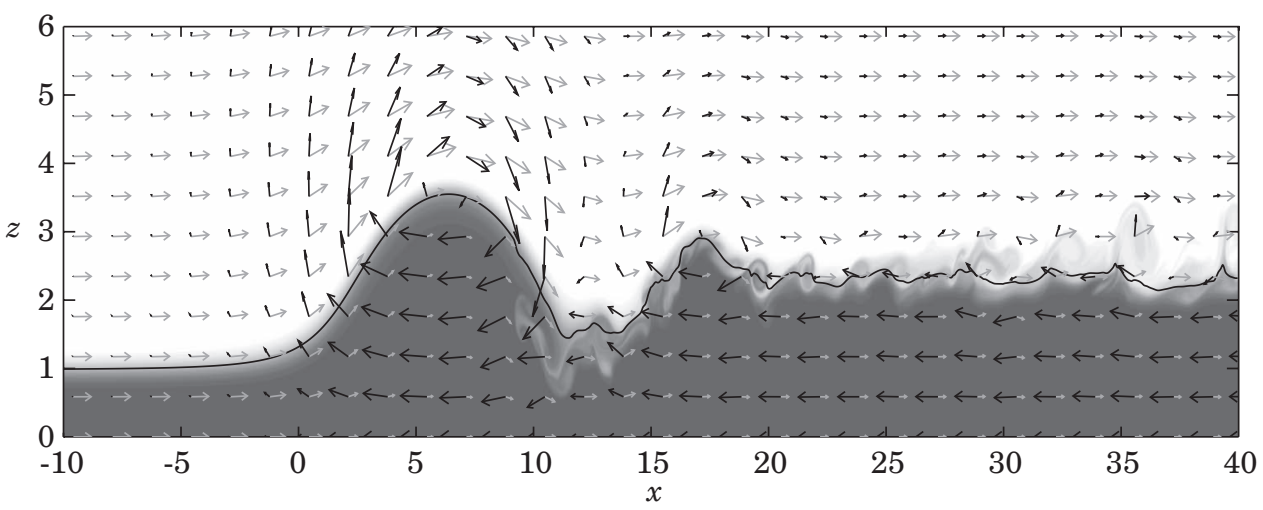

FIG. 4. Velocity vectors overlaid on the density field for a simulated internal bore at time $t=32$. The black arrows show the velocity field in the laboratory reference frame of the simulation, and the gray arrows show the velocity field in a reference frame moving with the front of the bore (scaled differently). In this simulation, $R=2.27, r=0.1, \gamma=0.4, R e=3500$, and $S c=1$. The velocity field is shown only at every 60th point in $x$ and 20th point in $z$. Here, $x=0$ corresponds to the front of the bore. The black line represents the local current height.

by the addition of the third dimension (they differed by $1 \%$ or less). They also found that twodimensional simulations correctly reproduced trends in three-dimensional parameter studies of turbulent mixing and non-hydrostatic effects. The only inconsistency found between two- and threedimensional simulations of internal bores is in the relative magnitudes of viscous dissipation and the viscous diffusion of momentum, but these two terms are of a smaller magnitude than any energy loss mechanism discussed later in this paper. We therefore feel confident in our decision to employ two-dimensional simulations.

Figure 4 shows the density and velocity fields in an example simulation. The black arrows show the velocity field in the reference frame of the simulation-a laboratory reference frame. But to compare our simulations with the two-layer analytical models, we must calculate the bore's front velocity and change reference frames into one moving with the front of the bore. Our method for computing the front velocity requires that we first compute the local height of the bore, $h(x)$, which we define as the position of the interface if we freeze the fluid's velocity field and allow the more dense, lower layer fluid to settle. Mathematically, this is expressed as

$$
h(x)=\int_{0}^{H} \frac{\rho-\gamma}{1-\gamma} \mathrm{d} z .
$$

With the local height known, we define the position of the front as the first $x$-location where the height exceeds some critical value, $h_{c}$, which we define as

$$
h_{c}=\frac{h_{0}-h_{a}}{4}+h_{a} .
$$

We track the front's position and differentiate it with respect to time to calculate the front velocity. Figure 5 shows that although the calculated position of the front is sensitive to our choice of $h_{c}$, the front velocity is not as long as the value of $h_{c}$ is reasonable (i.e., not greater than $h_{f}$ ).

Re-examining the velocity field in a reference frame moving with the front of the bore shows that the slip boundaries in our simulation produce a nearly uniform and horizontal velocity in each layer, except near the interface.

The other important parameter that cannot be specified a priori is the average current height, $R$. To calculate $R$, we take the mean value of $h(x)$ in a region that begins well downstream of the front of the bore and ends before the rarefaction wave propagating back into the initial fluid reservoir. For the bore in Figure 4, this region extends from $x=25$ to $x=40$.

The methods we use to calculate $u_{f}$ and $R$ are not the only ones available. Borden et al. ${ }^{15}$ explored two methods for determining the front location and four methods for calculating $R$. The methods described above are the most accurate and robust of the methods they investigated. 


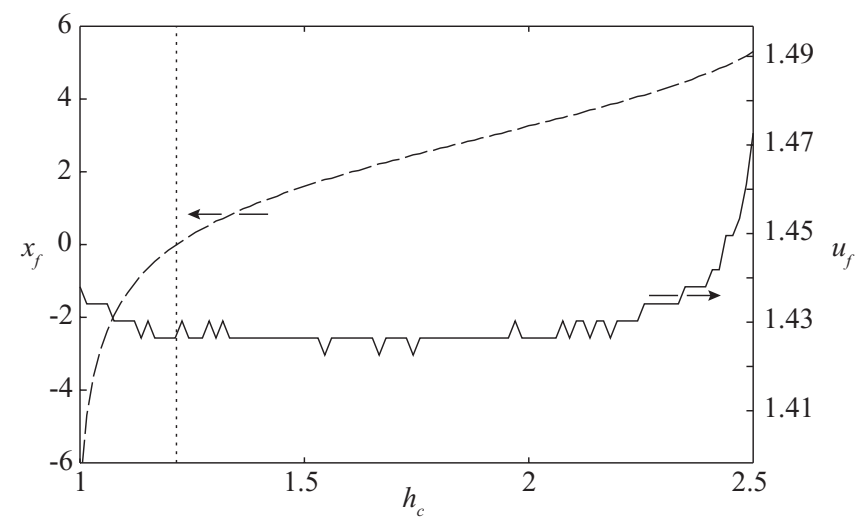

FIG. 5. Computed front position (dashed line) and front velocity (solid line) at $t=32$ as a function of $h_{c}$. The vertical dotted line denotes the critical height given by Eq. (29). In this simulation, $R=1.9, r=0.1, \gamma=0.5, R e=3500$, and $S c=1$.

The dependence of the front velocity on the density ratio is shown in Figure 6 for $R=1.9$, $r=0.1, R e=3500$, and $S c=1$. Our simulations predict that as the density ratio of a bore decreases, it propagates faster. Furthermore, whether or not the kinematic or dynamic viscosity is constant in the domain does not noticeably affect the front velocity for $\gamma>0.5$. For $\gamma<0.5$, bores with a constant kinematic viscosity propagate faster than ones with a constant dynamic viscosity, and the difference increases at even smaller density ratios. We were unable to simulate bores with $\gamma<0.2$ because the necessary grid resolution excessively increased the simulations' runtime, but we believe it is reasonable to conclude that the front velocities are converging to the correct singlelayer value. For the remainder of this investigation, we will focus on simulations with a constant dynamic viscosity, but will discuss the implications of a viscosity ratio in our concluding remarks.

For values of $\gamma$ close to one, our simulated bores propagate more slowly than both WS and KRS predict they should. According to the LC relation plotted in Figure 2, we therefore expect a global loss of energy, a loss of energy in the upper layer, and a gain of energy in the lower layer. This was confirmed by Borden et al. ${ }^{15}$ For density ratios below $\gamma \approx 0.5$, however, the simulated front velocities become larger than KRS as they tend toward the single-layer value, and must therefore lose energy across the lower layer of the bore. As we now attempt to model the change in energy flux across the bore, we must remember this sign change at $\gamma \approx 0.5$ as a phenomenon that a reasonable model should reproduce.

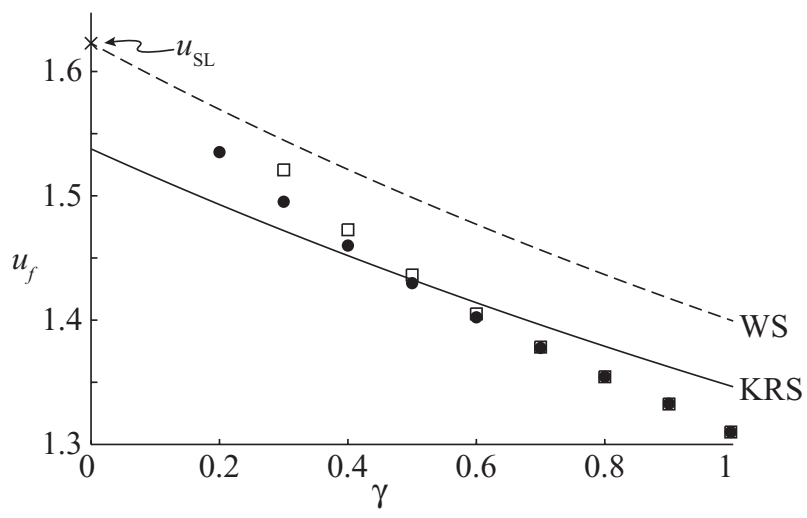

FIG. 6. Calculated front velocities in numerical simulations of internal bores plotted as a function of the density ratio for a constant kinematic $(\square)$, and dynamic $(\bullet)$ viscosity for $R=1.9, r=0.1, R e=3500$, and $S c=1$. The dashed line shows the prediction of the non-Boussinesq extension of the WS model (Eq. (10)), the solid line shows the prediction of KRS (Eq. (11)), and the " $\times$ " denotes the front velocity predicted by a single-layer hydraulic model. 


\section{MECHANISMS FOR ENERGY LOSS AND TRANSFER}

A closed form, two-layer non-Boussinesq model requires an expression that relates the gain or loss of energy across one layer to the bore's flow parameters. But exactly what constitutes such a change in energy is ambiguous. Eqs. (6) and (7), part of the non-Boussinesq LC relation, describe how the flux of energy changes across each layer of an ideal two-layer bore, and summing these two equations describes how the energy flux changes over a global control volume. If an ideal two-layer bore could conserve energy, $\dot{E}_{u}+\dot{E}_{l}$ would equal zero, and the sum of Eqs. (6) and (7) would become

$$
\begin{aligned}
\Phi_{\text {ideal }}= & \frac{1}{2} \rho_{2} g U\left(H^{2}-h_{a}^{2}\right)+\frac{1}{2} \rho_{2} g U\left(H-h_{a}\right)^{2}+p_{t l} U H+\rho_{1} g U h_{a}^{2} \\
& +\rho_{2} g U\left(H-h_{a}\right) h_{a}-\frac{1}{2} \rho_{2} g U_{2}\left(H^{2}-h_{f}^{2}\right)-\frac{1}{2} \rho_{2} g U_{2}\left(H-h_{f}\right)^{2} \\
& -p_{t r} U_{2}\left(H-h_{f}\right)-\rho_{1} g U_{1} h_{f}^{2}-p_{t r} U_{1} h_{f}-\rho_{2} g U_{1}\left(H-h_{f}\right) h_{f},
\end{aligned}
$$

where $\Phi=\int E \boldsymbol{u} \cdot \mathrm{d} \boldsymbol{A}$ is the total change in the flux of kinetic energy. Equation (30) shows that across an energy conserving bore, the flux of kinetic energy decreases as it is reversibly converted into potential and pressure energy. In arriving at this energy balance, we have made the following assumptions: that the hydrostatic assumption is valid, that the flow is inviscid, that the flow in each layer is uniform and horizontal, and that the flow is at a steady state. Without these assumptions, the energy balance becomes

$$
\Phi_{\text {real }}=\underbrace{\int_{V} \rho \boldsymbol{g} \cdot \boldsymbol{u} \mathrm{d} V}_{\text {(a) }}+\underbrace{\oint p \boldsymbol{u} \cdot \mathrm{d} \boldsymbol{A}}_{\text {(b) }}+\underbrace{\oint 2 R e^{-1} u_{i} S_{i j} \mathrm{~d} A_{j}}_{\text {(c) }}+\underbrace{\int_{V} \dot{\mathcal{D}} \mathrm{d} V}_{\text {(d) }}-\underbrace{\frac{\partial}{\partial t} \int_{V} E \mathrm{~d} V}_{\text {(e) }}
$$

where $\dot{\mathcal{D}}$ is the local rate of viscous dissipation and $E=(1 / 2) u_{i}^{2}$ is the local kinetic energy. From left to right, the terms in Eq. (31) represent:

(a) the work performed by body forces;

(b) the work performed by pressure forces;

(c) the viscous diffusion of momentum (or, equivalently, the work performed by viscous stresses);

(d) the viscous loss of kinetic energy to heat;

(e) the time rate of change of kinetic energy within the control volume.

Because of the additional terms in Eq. (31) compared to Eq. (30), the actual change in the flux of kinetic energy across a bore may be different than the change we expect based on the analytical control volume momentum balance (Eq. (30)). We define the difference between these two quantities, $\Phi_{\text {real }}-\Phi_{\text {ideal }}$, as the loss, or gain, of energy across a bore.

According to our definition, any mechanism which can alter the flux of kinetic energy and is not captured by the two-layer ideal energy balance, even if it is reversible, is considered a gain or loss of energy. Borden et al. ${ }^{15}$ explored three possible mechanisms: viscous diffusion and dissipation of momentum, potential modeling errors caused by invoking the hydrostatic assumption, and turbulent mixing at the interface between the upper and lower layers. They found that turbulent mixing has by far the greatest impact on the evolution of energy across a bore. By incorporating turbulent mixing into their analytical framework, they were able to derive an improved model for Boussinesq internal bores. We now extend that model to non-Boussinesq bores.

\section{A. Turbulent mixing}

To understand how turbulent mixing can affect the energy flux across an internal bore, consider the sketch of a a two-layer bore with an ideal density distribution in Figure 7. At cross-section A-A, near the front of the bore, relatively little mixing has occurred and the layer interface is sharp. At cross-section B-B the density profile is altered because of mixing at the interface caused by shear. For an idealized linear density profile, the potential energy at cross-section B-B is $\delta^{2} /[24(1-\gamma)]$ 

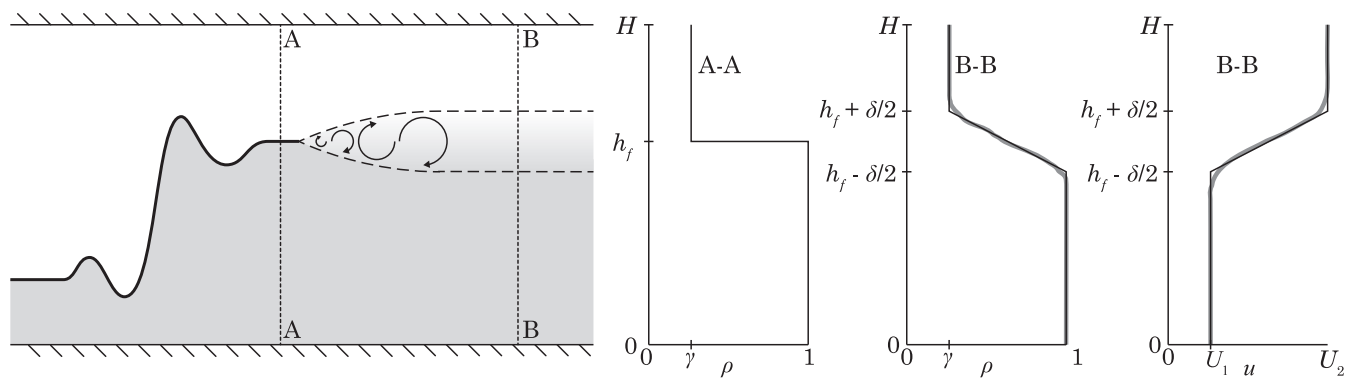

FIG. 7. The effect of turbulent mixing on the density stratification and velocity profile downstream of an internal bore. In the right two graphs, the thin black lines represent the ideal stairstep and linear density and velocity profiles at cross-sections A-A and B-B, and the thick grey lines represents the actual profiles from a simulation.

greater than at A-A, where $\delta$ is the thickness of the mixed layer. Because the total flux of potential energy increases as the mixing layer widens downstream, the flux of kinetic energy decreases. In a homogeneous shear layer, the thickness $\delta$ increases linearly with downstream distance, but in a stratified layer, $\delta$ asymptotes to a steady value. Turner ${ }^{23}$ was able to derive an expression for a stratified shear layer's maximum thickness by applying conservation of mass and conservation of momentum, equating the decrease in kinetic energy flux with the increase in potential energy flux, and assuming a linear profile for the density and velocity within the shear layer. His expression is

$$
\delta_{\max }=2 \gamma(\Delta U)^{2},
$$

where $\Delta U=U_{2}-U_{1}$. But Turner ${ }^{23}$ observed that the actual thickness of experimental stratified shear layers was much smaller than his theoretical prediction, and he attributed the difference to viscous losses and molecular diffusion. He therefore argued that Eq. (32) should be adjusted with a scaling parameter $f$ that could depend on the Reynolds and Schmidt numbers.

We derive an analytical model of an internal bore that includes mixing by taking the difference in the kinetic energy flux at cross-sections A-A and B-B, and combining that result with either Eq. (8) or (9) (the LC relation). We begin by calculating the energy change from mixing in the lower layer, $\dot{e}_{l}$, but will later re-derive an expression based on $\dot{e}_{u}$ to see if the choice of layer affects the results.

In the lower layer, the kinetic energy flux at both cross-sections for $z<h_{f}-\delta / 2$ is identical. The energy flux only differs over the range $h_{f}-\delta / 2 \leq z \leq h_{f}$. If we assume a linear velocity and density profile over this region in the fully developed shear layer (a common and good assumption, as seen in Figure 7), the kinetic energy fluxes in this region of the lower layer are given as

$$
\begin{aligned}
& \Phi_{l}(x=A A)=\frac{1}{2} \int_{0}^{\delta / 2} U_{1}^{3} \mathrm{~d} z^{*}, \\
& \Phi_{l}(x=B B)=\frac{1}{2} \int_{0}^{\delta / 2}\left(1+(\gamma-1) \frac{z^{*}}{\delta}\right)\left(U_{1}+\Delta U \frac{z^{*}}{\delta}\right)^{3} \mathrm{~d} z^{*},
\end{aligned}
$$

where $z^{*}=0$ corresponds to the lower edge of the fully developed shear layer. The apparent energy change in the lower layer is the difference between these two fluxes. Integrating and taking the difference, we are left with

$$
\begin{aligned}
\dot{e}_{l}= & \frac{\delta}{640}\left[120 U_{1}^{2} \Delta U+40 U_{1} \Delta U^{2}+5 \Delta U^{3}\right. \\
& \left.+(\gamma-1)\left(U_{1}^{3}+40 U_{1}^{2} \Delta U+15 U_{1} \Delta U^{2}+2 \Delta U^{3}\right)\right] .
\end{aligned}
$$

The limit of integration $\delta$ is given by Eq. (32). Introducing this scaling into Eq. (35), and applying an unmixed conservation of mass as stated in Eq. (1) to the result, provides the following expression for the energy change in the lower layer due to turbulent mixing:

$$
\dot{e}_{l}=\frac{\beta_{l}(R-1)}{R^{2}(1-R r)} u_{f}^{5},
$$


where

$$
\begin{aligned}
\beta_{l}= & \frac{f \gamma(R-1)}{R^{3}(1-R r)^{4}}\left\{120(R-1)(1-R r)^{2}+40(R-1)^{2}(1-R r)+5(R-1)^{3}\right. \\
& \left.+(\gamma-1)\left[40(1-R r)^{3}+40(R-1)(1-R r)^{2}+15(R-1)^{2}(1-R r)+2(R-1)^{3}\right]\right\},
\end{aligned}
$$

and $f$ is Turner's scaling coefficient. Borden et al. ${ }^{15}$ used an expression of $f=0.5 P e^{-1 / 2}$, which, although based on the assumption of a Blasius concentration boundary layer, was able to accurately model the font velocity's dependence on the Schmidt number in the Boussinesq case. We will use their expression here as well. To obtain the bore's front velocity, we combine Eq. (36) with the LC relation stated in Eq. (9) and get

$$
u_{f}^{2}=\frac{-b+\left(b^{2}-4 a c\right)^{1 / 2}}{2 a}
$$

where

$$
\begin{aligned}
& a=2 \beta_{l}, \\
& b=2 R^{2} r^{2}(1-\gamma)+R^{2} r(2 \gamma-1)-3 R r+R+1, \\
& c=R^{2}(R r+r-2)(1-R r) .
\end{aligned}
$$

The front velocity in Eq. (37) was derived by comparing the pre- and post-mixing energy fluxes in the lower layer to get an expression for $\dot{e}_{l}$, which we substituted into Eq. (9). A similar model can be obtained by finding an expression for $\dot{e}_{u}$, and substituting that into Eq. (8). Following a similar approach, we find that

$$
\dot{e}_{u}=\frac{\beta_{u}(R-1)(1-r)}{R(1-R r)^{2}} u_{f}^{5},
$$

where

$$
\begin{aligned}
\beta_{u} & =\frac{f \gamma(R-1)}{R^{4}(1-R r)^{3}(1-r)}\left\{\gamma\left[-120 R^{2}(R-1)(1-r)^{2}+40 R(R-1)^{2}(1-r)-5(R-1)^{3}\right]\right. \\
& \left.-(\gamma-1)\left[40 R^{3}(1-r)^{3}-40 R^{2}(R-1)(1-r)^{2}+15 R(R-1)^{2}(1-r)-2(R-1)^{3}\right]\right\} .
\end{aligned}
$$

When this expression is substituted into Eq. (8), the coefficients in Eq. (37) become

$$
\begin{aligned}
& a=2 \beta_{u}, \\
& b=2 R^{2} r^{2}(\gamma-1)-\gamma R^{2} r+R r(4-\gamma)-2, \\
& c=R(R+1)(1-R r)^{2} .
\end{aligned}
$$

Hereafter, we refer to the model based on mixing in the lower layer as the mixing- $\dot{e}_{l}$ model, and the model based on mixing in the upper layer as the mixing- $\dot{e}_{u}$ model. Comparing the front velocities predicted by these two models with numerical simulations in Figure 8, we see that in the Boussinesq case, both the mixing- $\dot{e}_{l}$ and mixing- $\dot{e}_{u}$ models predict the same front velocity, one which accurately describes the simulations' results. As the density ratio decreases, however, the density stratification at the interface becomes stronger and it suppresses turbulence, which in turn thins the shear layer. Mathematically, this can be seen in Eq. (32), where the thickness $\delta$ scales linearly on the density ratio $\gamma$. Because the mixing layer thickness goes to zero in the limit of large density differences, the mixing- $\dot{e}_{u}$ and mixing- $\dot{e}_{l}$ models diverge and tend to the WS and KRS velocities.

A comparison of the energy change due to turbulent mixing in the models and in the simulations (Figure 9) shows that the energy change in the lower layer due to turbulent mixing is better predicted by the mixing- $\dot{e}_{l}$ model, but the mixing- $\dot{e}_{u}$ model reproduces the single-layer limit for $\gamma=0$. Neither fit is ideal and the simulations' energy change due to mixing does not tend to the single layer limit, both of which suggests that although turbulent mixing is the dominant mechanism for energy loss and transfer across Boussinesq bores, some other energy transfer mechanism must become important 


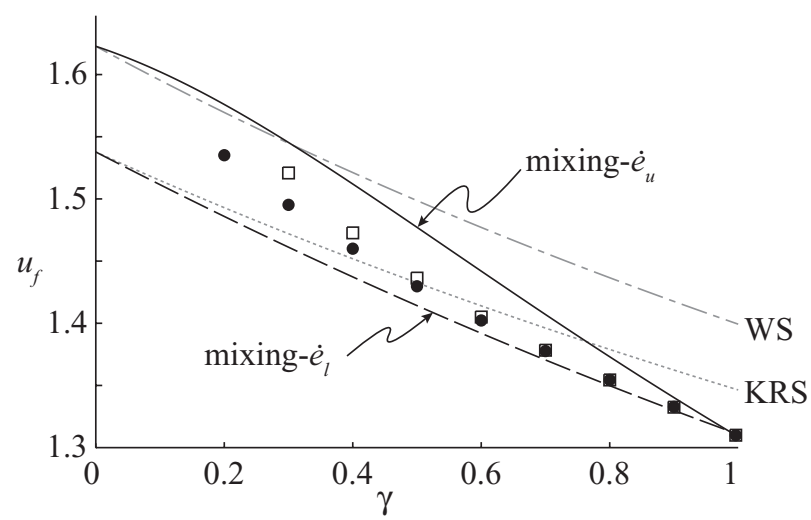

FIG. 8. Black lines represent predictions from the mixing- $\dot{e}_{l}$ (dashed line) and mixing- $\dot{e}_{u}$ (solid line) models for the front velocity as a function of density ratio for $R=1.9, r=0.1, R e=3500$, and $S c=1$. The symbols represent measurements from simulations for a constant kinematic $(\square)$, and dynamic $(\bullet)$ viscosity. The dotted gray line shows the prediction of the KRS model, and the dash-dotted gray line shows the prediction of WS.

as we decrease the density ratio. We believe the new mechanism is the generation of undular waves at the front of non-Boussinesq bores, which we now discuss.

\section{B. Undular wave generation}

The classic single-layer model of hydraulic jumps developed by Rayleigh ${ }^{24}$ predicts the loss of energy across a bore given by Eq. (17). Most researchers attributed the energy loss to "friction" associated with the generation and breaking of waves at the bore's front, but Favre ${ }^{25}$ noticed that for bores with $R<1.28$, the waves generated at the front of the bore do not break. Lemoine ${ }^{26}$ argued that under these circumstances, the loss of energy predicted by the single-layer model occurs through the generation and radiation of waves, not friction.

Lighthill ${ }^{27}$ explains thoroughly the reason that bores radiate waves. To summarize, Fourier analysis of the surface shape of the front of a hydraulic jump shows that it contains sinusoidal components with a short wavelength compared to the jump's depth. These waves propagate more slowly than the long-wave velocity relative to the front of the bore and therefore fall behind, radiating energy away from the front of the bore.

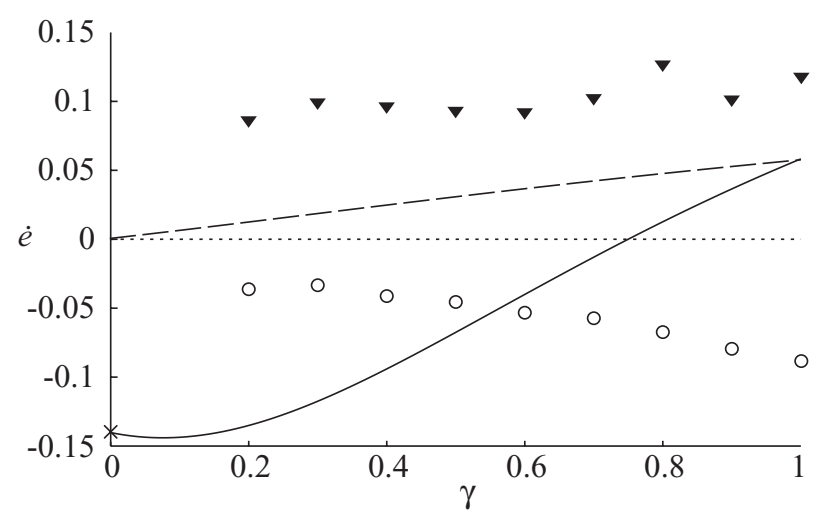

FIG. 9. Global (०) and lower layer ( $\mathbf{\nabla})$ energy change due to turbulent mixing in our simulations as a function of the density ratio for $R=1.9, r=0.1, R e=3500, S c=1$, and $\mu_{1}=\mu_{2}$. The lines represent the predicted energy change in the lower layer from the mixing- $\dot{e}_{l}$ (dashed line) and mixing- $\dot{e}_{u}$ (solid line) models. The " $\times$ " represents the energy change in the lower layer predicted by the single-layer hydraulic model. 

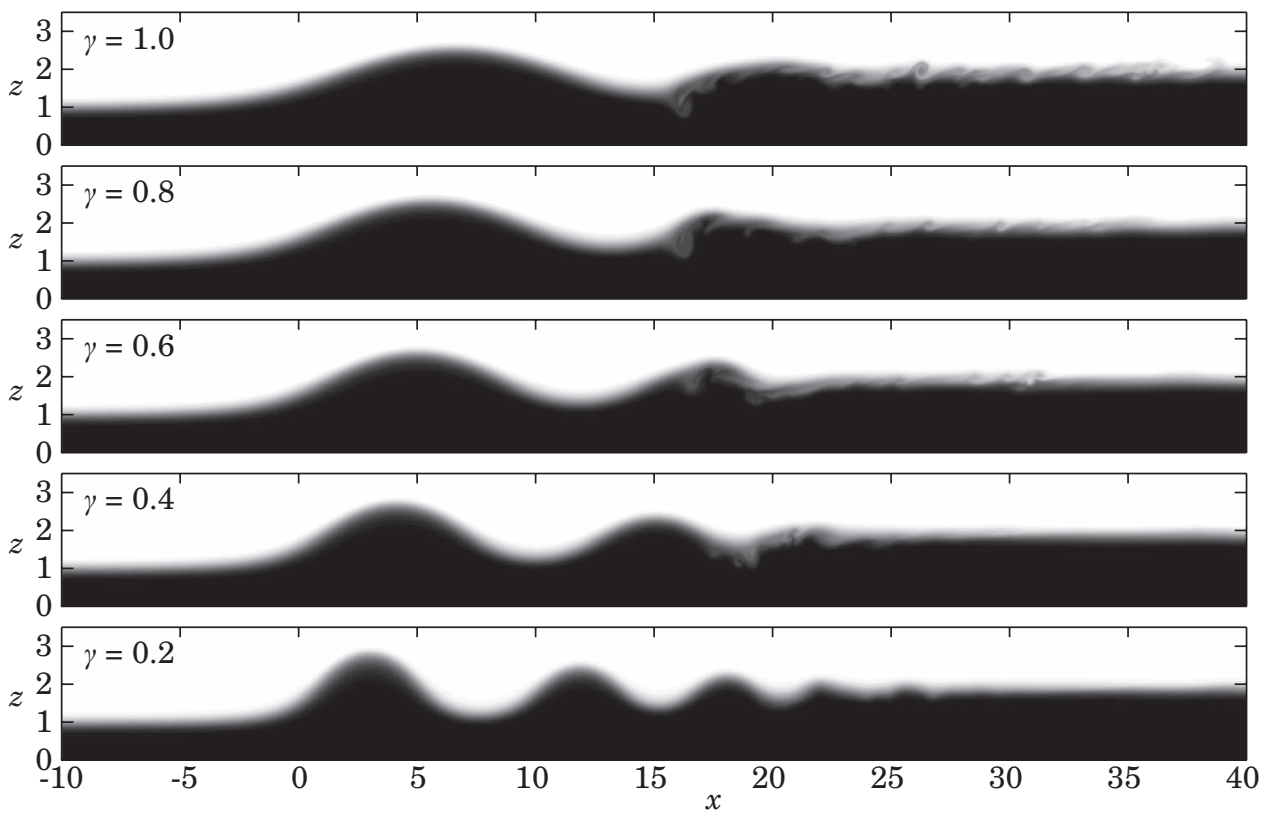

FIG. 10. Density profile of five non-Boussinesq bores with different density ratios shown at $t=32$ for $R=1.9, r=0.1$, $R e=3500, S c=1$, and $\mu_{1}=\mu_{2}$. As the density ratio decreases, the front of the bore becomes more undular.

When the size of a single-layer bore surpasses a critical value, it no longer radiates waves. Researchers still debate the exact limit—values ranging between $1.7 \leq R_{\text {crit }} \leq 4.5$ have been observed-but a generally agreed upon value seems to be $R_{\text {crit }}=2.7$ (Ref. 28). Bores smaller than $R_{\text {crit }}$ will dissipate some of their energy through the radiation of waves.

For Boussinesq internal bores, the value of $R_{\text {crit }}$ depends on the channel depth and is smaller than for single-layer bores. Borden et al. ${ }^{15}$ observed that for $r=0.1$, the upper limit on wave generation was $R_{\text {crit }} \approx 1.5$. Because most of their simulated bores were larger than $R_{\text {crit }}$, they ignored wave radiation in their model. They did, however, notice discrepancies between their model and simulations at small values of $R$ and attributed the difference to wave radiation.

As the density ratio decreases, we expect $R_{\text {crit }}$ to increase to its single-layer value. Figure 10 compares a Boussinesq internal bore with several non-Boussinesq bores at different density ratios. Because the Boussinesq bore is larger than the critical value of $R_{\text {crit }} \approx 1.5$, there are no waves radiated from the front. There is a single wave that travels ahead of the bore, but this wave does not grow in time, and therefore does not act as an energy sink. Most of the energy lost across the bore is because of turbulent mixing. As the density ratio decreases to where the value of $R_{\text {crit }}$ is larger, turbulent mixing is suppressed and the front becomes undular. Furthermore, several height profiles of the $\gamma=0.2$ bore, plotted in Figure 11(a), show that the number and amplitude of these waves grow in time.

The energy of a gravity wave is split evenly between kinetic and potential components. We can therefore calculate the total energy in the undular waves at a bore's front by calculating the wave's excess potential energy and multiplying by two. If we define the position of the interface separating the upper and lower layers as $\zeta(x, t)$, where $\zeta=0$ corresponds to an unperturbed base state, the excess potential energy of an internal wave works out to be $\int(1 / 2) \zeta(x, t)^{2} \mathrm{~d} x$, and the total wave energy is $E_{\text {wave }}(t)=\int \zeta(x, t)^{2} \mathrm{~d} x$. We define the unperturbed base state of our simulations to be

$$
h(\zeta=0)= \begin{cases}1 & \text { for } x \leq x_{f} \\ R & \text { for } x>x_{f}\end{cases}
$$



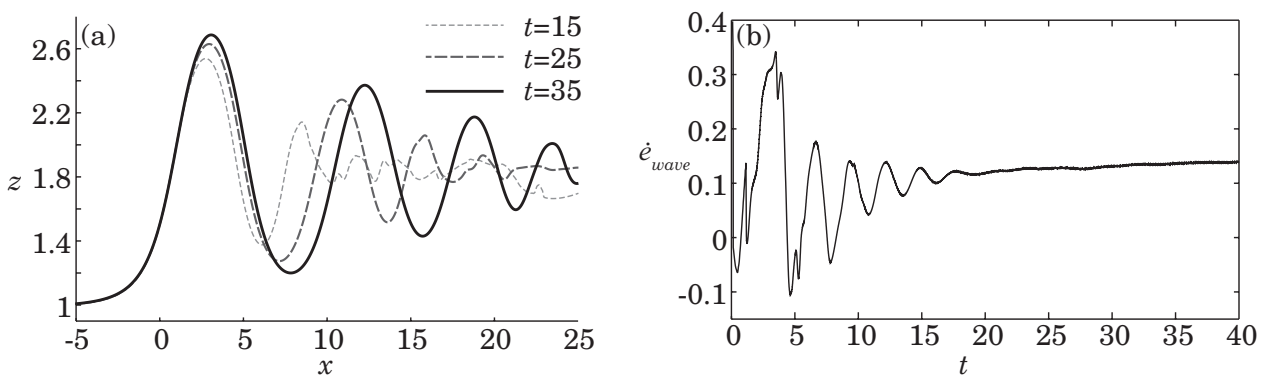

FIG. 11. (a) Height profile of a non-Boussinesq bore $(\gamma=0.2)$ at three different times for $R=1.9, r=0.1, R e=3500$, and $S c=1$. The waves at the front of the bore grow in time, both in amplitude and in number, and are radiated away from the front. (b) Rate of change of wave energy - computed with Eq. (40) - as a function of time over the course of the simulation. After an initial transient period, the rate of change of wave energy reaches a quasi-steady value.

The rate of energy change to wave radiation is the time derivative of the total wave energy. We calculate it as

$$
\dot{e}_{\text {wave }}(t)=\frac{\partial}{\partial t} \int_{x_{1}}^{x_{2}} \zeta(x, t)^{2} \mathrm{~d} x
$$

where $x_{1}$ is some location well ahead of the bore and $x_{2}$ is a location downstream after the end of the undular waves but before the rarefaction wave in our simulation. In the extreme non-Boussinesq limit, $\gamma=0, \dot{e}_{\text {wave }}$ is entirely confined to the lower layer. In the Boussinesq limit, $\dot{e}_{\text {wave }}$ is split evenly between the two layers. The fraction of $\dot{e}_{\text {wave }}$ in each layer varies linearly between these two limits for intermediate values of $\gamma$. Just as with other global flow properties such as front velocity and current height, $\dot{e}_{\text {wave }}$ attains a quasi-steady value after the initial transient period of the simulation. This can be seen in Figure 11(b), which tracks the time rate of change of the wave energy over the course of a simulation.

In our simulations, we observe that the global rate of energy loss to wave radiation increases with decreasing density ratio (Figure 12(a)). This finding is consistent with an argument that $R_{\text {crit }}$ increases as the density ratio decreases. Furthermore, we see that the magnitudes of the rates of energy change due to wave radiation are on the same order as the change in energy flux due to mixing, plotted earlier in Figure 9. Both effects must be important.

The sum of the energy change due to mixing and wave radiation is plotted for several simulations in Figure 12(b). The total amount of energy loss in both layers does not appear to vary strongly as a function of the density ratio, but there is a strong dependence in each layer separately. For density ratios close to one, the energy change in the lower layer is positive, but it becomes negative once the
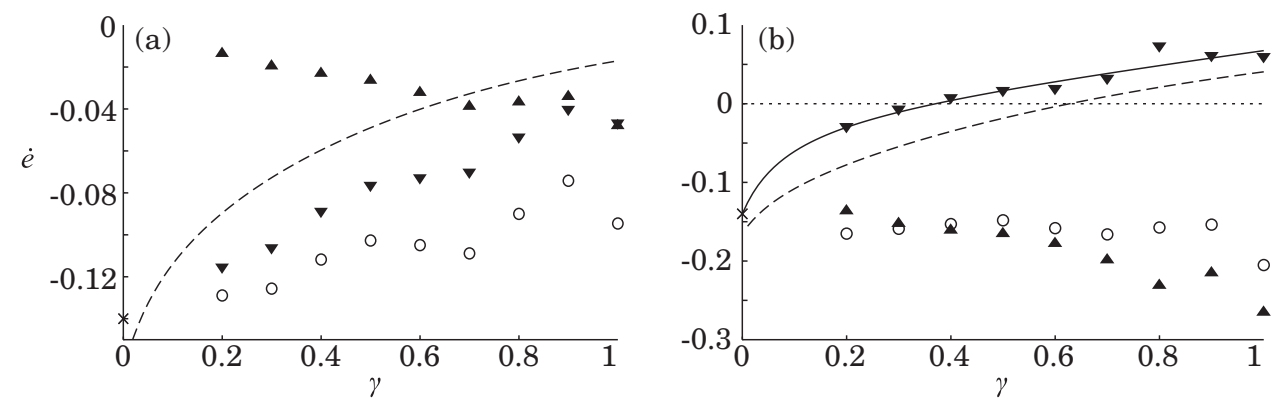

FIG. 12. (a) Rate of energy change due to wave radiation in the upper ( $\mathbf{\Lambda})$, the lower $(\mathbf{\nabla})$, and both (o) layers in our simulations as a function of density ratio for $R=1.9, r=0.1, R e=3500, S c=1$, and $\mu_{1}=\mu_{2}$. The dashed line is the prediction of Eq. (41) scaled by 0.5. (b) Measured energy change in each layer, and globally, due to both turbulent mixing and wave radiation. The solid black line is a rational function fit to the lower layer simulation data and to the single layer value $(x)$. The dashed line is the sum of Eqs. (36) and (41). In both figures, $\times$ represents the single-layer energy loss. 
(a) $R=1.9$
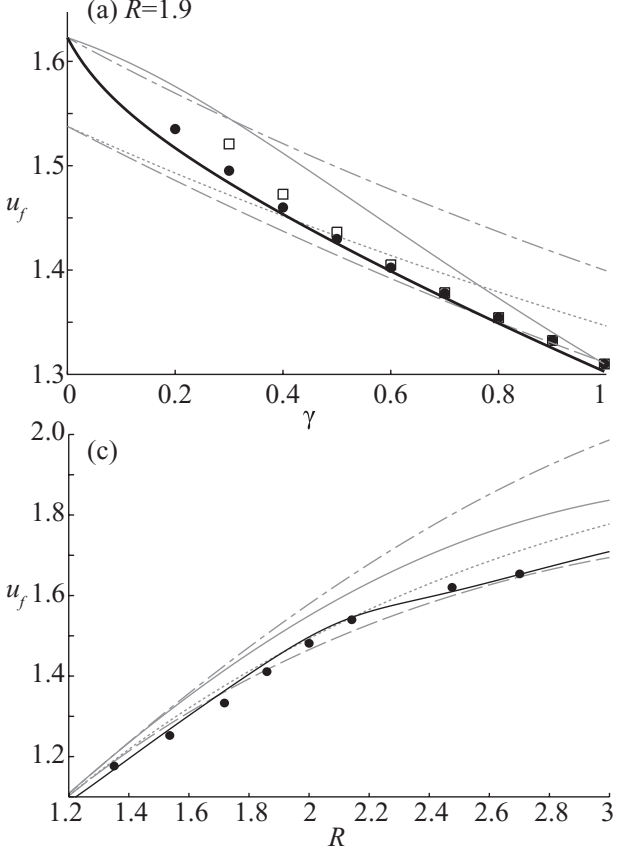

(b) $R=2.35$

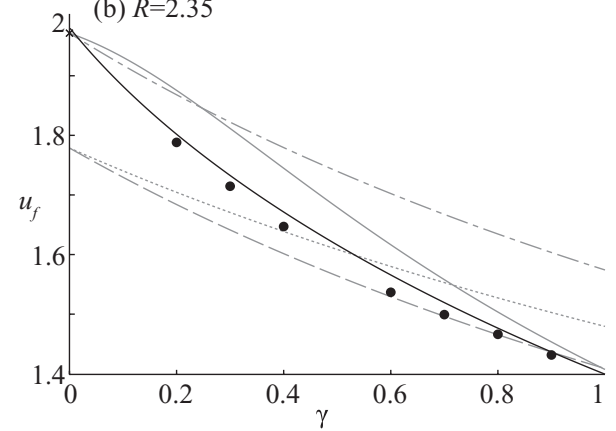

$-\cdots--W S$

$K R S$

mixing- $\dot{e}_{\vec{l}}$

mixing-e

mixing $-\dot{e}_{l}+$ waves

FIG. 13. (a) The thick black line represents the predicted front velocity obtained by fitting a curve to the energy change in the lower layer due to mixing and wave radiation and applying the result to Eq. (8) for $R=1.9, r=0.1, R e=3500$, and $S c=1$. The symbols represent measurements from simulations for a constant kinematic $(\square)$, and dynamic $(\bullet)$ viscosity. Gray lines represent the predictions of the mixing- $\dot{e}_{l}$ model (dashed line), mixing- $\dot{e}_{u}$ model (solid line), KRS model (dotted line), and WS model (dash-dotted line). (b) Same as frame (a), but with $\mathrm{R}=2.35$ instead. (c) Same as (a), but with $R$ varying as $\gamma=0.5$ is held constant.

density ratio decreases beyond $\gamma=0.4$. The crossover point corresponds nicely to the density ratio at which we observe our simulation's front velocities increasing over KRS.

A model which takes account of wave radiation and turbulent mixing would be an improvement from the mixing- $\dot{e}_{l}$ and mixing- $\dot{e}_{u}$ models. This becomes evident if we fit a curve to the observed energy change due to these two effects in the lower layer, plotted in Figure 12(b), and combine it with the LC relation to predict front velocity. A comparison of this new model with our simulations front velocities (Figure 13(a)) shows a large improvement. We accurately capture the density ratio at which the front velocities decrease below KRS, and the model tends towards the single-layer front velocity for small density ratios. At these smaller density ratios, the new model better approximates simulations with a constant dynamic viscosity, but that is only because it is based upon a curve fit to data from simulations that also have a constant dynamic viscosity.

Unfortunately, predicting the amount of energy a non-Boussinesq internal bore radiates through waves is not as straightforward as calculating the energy change due to turbulent mixing. Benjamin and Lighthill ${ }^{29}$ found that if the waves radiated by a bore were conoidal, the maximum rate of energy loss across a single-layer bore is

$$
\dot{e}_{\max }=\frac{1}{2} R^{2}\left[1+\frac{5}{2} u_{f}^{2}-\frac{1}{8} u_{f}^{4}-\frac{1}{8} u_{f}\left(8+u_{f}^{2}\right)^{3 / 2}\right] .
$$

But their equation has several drawbacks which limit its usefulness in the present context. First, it neglects the presence of the upper layer which limits its application to small values of $\gamma$. Second, the theory upon which it is based is only valid if the wavelength of the radiated waves is large compared to the fluid depth. For values of $R>1.3$, the observed wavelengths are too short, compared to the fluid depth, for the theory to be accurate..$^{29}$ Finally, Benjamin and Lighthill ${ }^{29}$ proved that radiated waves cannot be responsible for the entirety of the energy loss predicted by Eq. (41). The equation represents an unobtainable upper bound, and some energy must be lost by another mechanism, which they assumed to be frictional dissipation. 
Despite these limitations and assumptions, we see in Figure 12(a) that Eq. (41) is modestly accurate at predicting the energy change in the lower layer because of radiated waves when we arbitrarily scale it by 0.5 (because the equation represents an upper limit). Furthermore, summing a scaled Eq. (41) with the expression we derived for the energy change in the lower layer due to mixing (Eq. (36)) produces a reasonable fit to the measured effect of mixing and waves on the lower layer of our simulation.

\section{CONCLUSIONS}

In this investigation, we used highly resolved two-dimensional direct numerical simulations to evaluate two-layer hydrostatic models for non-Boussinesq internal bores. Our goal was to search for mechanisms that can alter the flux of kinetic energy across a bore. Following the Boussinesq work of Borden et al. ${ }^{15}$ we investigated the effect of turbulent mixing at the interface between the upper and lower layers. For nearly Boussinesq bores, turbulent mixing has a strong effect on the energy flux, and therefore front velocity. But as the density difference across the layer's interface becomes greater, the turbulent mixing is suppressed. In these cases, we found that the energy lost to the generation of undular waves at the bore's front becomes important. An empirical fit to the measured energy change due to mixing and wave radiation, when combined with our non-Boussinesq adaptation of the LC relation, excellently predicts the velocity of the simulated non-Boussinesq bores as a function of the density ratio. We also considered an analytical expression derived by Benjamin and Lighthill ${ }^{29}$ which predicts, assuming large density differences and small bores, the maximum amount of energy that can be lost to wave radiation. Combining their equation with our expression for the energy change due to mixing predicts the total energy change in the lower layer reasonably well. For future work, a better fit could be obtained by re-deriving Benjamin and Lighthill's expression for an arbitrary density ratio.

Within this investigation, we examined the influence of the density ratio as it varied only for one fixed value of $R, r, R e$, and $S c$. But our findings extend to other parameter combinations, which can be seen in Figure 13(b). There, it can be seen that each model's relation to our simulation data holds for other values of $\mathrm{R}-$ in this case, $R=2.35$. Also, the influence of $R, r, R e$, and $S c$ on the propagation velocity and rate of energy change in each layer have been extensively examined in the Boussinesq case by Borden et al. ${ }^{15}$ We verified with a few simulations that the manner in which these parameters affect the front velocity is similar for non-Boussinesq bores as well (e.g., as in Figure 13(c)).

Finally, within this investigation, we were only able to simulate non-Boussinesq bores down to a density ratio of $\gamma=0.2$, and none of the bores we simulated were large enough for the leading wave to break. As the density ratio further decreases, one would expect the bores to behave more like single-layer bores, which can have turbulent fronts if they are sufficiently large. If the leading wave of a bore breaks, another energy loss mechanism becomes relevant, the viscous dissipation of momentum to heat. For future work, it would be interesting to simulate strongly non-Boussinesq bores that exhibit wave breaking to see how their front velocity is affected, and how the amount of breaking scales as a function of various parameters. If viscous effects become important, however, we expect that the ratio of the viscosities, as well as how the viscosity varies with concentration, will become important parameters as well.

\section{ACKNOWLEDGMENTS}

This research has been supported by National Science Foundation (NSF) Grant Nos. CBET0854338, CBET-1067847, and OCE-1061300. Our simulations were carried out at the Community Surface Dynamics Modeling System (CSDMS) high-performance computing facility at the University of Colorado in Boulder. We thank CSDMS director Professor James Syvitski and the technical staff at CSDMS for their support.

${ }^{1}$ J. E. Simpson, Gravity Currents, 2nd ed. (Cambridge University Press, 1997).

${ }^{2}$ W. H. Hager, Energy Dissipators and Hydraulic Jump (Kluwer Academic, Dordrecht, The Netherlands, 1992). 
${ }^{3}$ L. D. Landau and E. M. Lifshitz, Fluid Mechanics (Pergamon, Oxford, 1959).

${ }^{4}$ R. H. Clarke, R. K. Smith, and D. G. Reid, "The morning glory of the gulf of Carpentaria: an atmospheric undular bore," Mon. Weather Rev. 109, 1726-1750 (1981).

${ }^{5}$ R. M. Wakimoto and D. E. Kingsmill, "Structure of an atmospheric undular bore generated from colliding boundaries during CaPE," Mon. Weather Rev. 123, 1374-1393 (1995).

${ }^{6}$ S. P. Haase and R. K. Smith, "Morning glory wave clouds in Oklahoma: A case study," Mon. Weather Rev. 112, 2078-2089 (1984).

${ }^{7}$ J. J. Leichter, S. R. Wing, S. L. Miller, and M. W. Denny, "Pulsed delivery of subthermocline water to Conch Reef (Florida Keys) by internal tidal bores," Limnol. Oceanogr. 41, 1490-1501 (1996).

${ }^{8}$ E. G. Morozov, K. Trulsen, M. G. Velarde, and V. I. Vlasenko, "Internal tides in the Straight of Gibraltar," J. Phys. Oceanogr. 32, 3193-3206 (2002).

${ }^{9}$ P. Hosegood and H. van Haren, "Near-bed solibores over the continental slope in the Faeroe-Shetland Channel," Deep-Sea Res., Part II 51, 2943-2971 (2004).

${ }^{10}$ E. Gonzalez-Juez, E. Meiburg, and G. Constantinescu, "Gravity currents impinging on bottom-mounted square cylinders: Flow fields and associated forces," J. Fluid Mech. 631, 65-102 (2009).

${ }^{11}$ E. Gonzalez-Juez and E. Meiburg, "Shallow-water analysis of gravity-current flows past isolated obstacles," J. Fluid Mech. 635, 415-438 (2009).

${ }^{12}$ E. Gonzalez-Juez, E. Meiburg, T. Tokyay, and G. Constantinescu, "Gravity current flow past a circular cylinder: forces, wall shear stresses and implications for scour," J. Fluid Mech. 649, 69-102 (2010).

${ }^{13}$ I. R. Wood and J. E. Simpson, “Jumps in layered miscible fluids," J. Fluid Mech. 140, 329-342 (1984).

${ }^{14}$ J. B. Klemp, R. Rotunno, and W. C. Skamarock, "On the propagation of internal bores," J. Fluid Mech. 331, 81-106 (1997).

${ }^{15}$ Z. Borden, E. Meiburg, and G. Constantinescu, "Internal bores: An improved model via a detailed analysis of the energy budget," J. Fluid Mech. 703, 279-314 (2012).

${ }^{16}$ L. Yang, B. J. Azzopardi, A. Belghazi, and S. Nakanishi, "Phase separation of liquid-liquid two-phase flow at a T-junction," AIChE J. 52, 141-149 (2006).

${ }^{17}$ P. Roberts and S. Hibberd, “Internal hydraulic jumps at T-junctions," J. Fluid Mech. 314, 331-347 (1996).

${ }^{18}$ V. K. Birman, J. E. Martin, and E. Meiburg, "The non-Boussinesq lock-exchange problem. Part 2. High-resolution simulations," J. Fluid Mech. 537, 125-144 (2005).

${ }^{19}$ M. Ungarish, An Introduction to Gravity Currents and Intrusions (CRC, 2009).

${ }^{20} \mathrm{M}$. Ungarish, "Two-layer shallow-water dam-break solutions for non-Boussinesq gravity currents in a wide range of fractional depth," J. Fluid Mech. 625, 27-59 (2011).

${ }^{21}$ R. Rotunno, J. B. Klemp, G. H. Bryan, and D. J. Muraki, "Models of non-Boussinesq lock-exchange flow," J. Fluid Mech. 675, 1-27 (2011).

${ }^{22}$ M. Li and P. F. Cummins, "A note on hydraulic theory of internal bores," Dyn. Atmos. Oceans 28, 1-7 (1998).

${ }^{23}$ J. S. Turner, "Turbulent entrainment: the development of the entrainment assumption, and its application to geophysical flows," J. Fluid Mech. 173, 431-471 (1986).

${ }^{24}$ L. Rayleigh, "On the theory of long waves and bores," Proc. R. Soc. London, Ser. A 90, 324-328 (1914).

${ }^{25} \mathrm{H}$. Favre, Etude théorique et expérimentale des ondes de translation dans les canaux découverts (Dunod, Paris, 1932).

${ }^{26}$ R. Lemoine, "Sur les ondes positives de translation dans les canaux et sur le ressaut ondulé de faible amplitude," La Houille Blanche 2, 183-185 (1948).

${ }^{27}$ J. Lighthill, Waves in Fluids (Cambridge University Press, 1978).

${ }^{28}$ I. Ohtsu, Y. Yasuda, and H. Gotoh, "Hydraulic condition for undular-jump formations," J. Hydraul. Res. 39, 203-209 (2001).

${ }^{29}$ T. B. Benjamin and M. J. Lighthill, “On cnoidal waves and bores,” Proc. R. Soc. London, Ser. A 224, 448-460 (1954). 